\title{
Valores Normativos de la Escala Multidimensional de Autoeficacia Infantil para Población Argentina
}

\author{
Normative Data of Multidimensional Scale of Self-Efficacy for Children
}

\author{
Laura Beatriz Oros ${ }^{1}$
}

\section{Resumen}

Este artículo presenta la normalización de la Escala Multidimensional de Autoeficacia Infantil (EMA-i) para niños argentinos de 9-13 años de edad. La EMA-i fue diseñada con el propósito de detectar cuán capaces se consideran los niños para realizar con éxito diferentes actividades académicas, sociales y deportivas. La muestra de estudio estuvo compuesta por 1271 niños escolarizados, de ambos sexos, con una media de edad de 11.17 años $(\mathrm{DE}=1.07)$. Todos pertenecen a la clase media y fueron seleccionados de diferentes provincias y regiones de la República Argentina. Los resultados mostraron diferencias significativas entre los distintos sexos y edades por lo que se ofrecen los valores percentilares, las medias y desvíos de cada dimensión de la escala para cada grupo de niños.

Palabras clave: autoeficacia, niños, evaluación, valores normativos

\begin{abstract}
This article presents a standardized Children's Multidimensional Self-efficacy Scale for Argentine children aged 9-13 years. The CMSS was designed aiming at identifying how capable children are to successfully perform various academic, social and sporting activities. The standardization sample consisted of 1271 school children of both sexes, with a mean age of 11.17 years $(\mathrm{SD}=1.07)$. All of them were middle-class children and were selected from different provinces and regions of Argentina. The results showed significant differences among sexes and ages so that the percentile values, means and deviations of each dimension of the scale for each group of children are provided.
\end{abstract}

Keywords: self-efficacy, children, assessment, normative data

\footnotetext{
${ }^{1}$ Doctora en Psicología. Investigadora del CONICET-Universidad de la Cuenca del Plata; Universidad Adventista del Plata-ISAM. Avenida Rusia 410 (3315). Leandro N. Alem, Misiones, Argentina. Correo: lauraorosb@gmail.com 


\section{Introducción}

En el marco de la teoría social cognitiva, Bandura (1987) propuso el término autoeficacia para referirse al "juicio emitido sobre la propia capacidad para alcanzar un cierto nivel de ejecución" (p. 416). En otras palabras, se trata de la evaluación que realizamos sobre nuestra capacidad de realizar con éxito determinadas actividades o tareas.

Si bien es aceptado hablar de una autoeficacia general (Brenlla, Aranguren, Rossaro, \& Vázquez, 2010; Cid, Orellana, \& Barriga, 2010; Sanjuán, Pérez, \& Bermúdez, 2000), Bandura (1986) enfatiza que este sistema de creencias está ligado a áreas de funcionamiento específicas. A partir de esta idea, diferentes autores presentan el concepto de autoeficacia orientado a diversos ámbitos de actuación, como el social, el deportivo, el académico, el laboral, etc. Incluso dentro de cada uno de estos ámbitos, las creencias de autoeficacia pueden observarse de forma más particularizada, como cuando se analiza la autoeficacia académica para las matemáticas (Larson, Pesch, Surapaneni, Bonitz, Wu, \& Werbel, 2014; Usher \& Pajares, 2009), para el aprendizaje relacionado con las inteligencias múltiples (Pérez, Beltramino, \& Cupani, 2003; Pérez \& Medrano, 2007; Pérez \& Cupani, 2008); para la escritura y el rendimiento en Lengua (Pajares, 1996; Pérez, Medrano, Mattus, \& Ayllón, 2008), etc.

El modelo propuesto por Bandura (1997) afirma que las creencias de autoeficacia median la relación entre las capacidades reales y la conducta, siendo esenciales para explicar el comportamiento humano. Las personas que confían en sus propias capacidades y se sienten seguras de poder alcanzar sus metas tienden a encarar las situaciones complejas en lugar de evitarlas. Además, aumentan sus esfuerzos para el logro y desarrollan mayor persistencia ante las dificultades. De esta manera, la autoeficacia juega un rol preponderante para el funcionamiento humano, desempeñando un importante papel de autorregulación (Costa, 2012).

Según Flammer (1999) las creencias de autoeficacia no están presentes desde el nacimiento sino que se van desarrollando y consolidando a lo largo de cinco estadios o niveles evolutivos, iniciándose con las primeras experiencias de contingencia entre la conducta y el resultado, durante los primeros días de vida, hasta el surgimiento de los conceptos mentales de habilidad, esfuerzo, dificultad, etc., que se alcanzarían en torno a los 10 años, o antes, según el nivel madurativo de cada niño.

Estas creencias de eficacia personal se construyen en base a tres fuentes principales de información (Bandura, Pastorelli, Barbaranelli, \& Caprara, 1999): (a) las experiencias previas exitosas, (b) las experiencias vicarias y (c) la persuasión verbal o influencia social. Con respecto al contexto de relaciones, se puede pensar que durante la niñez y adolescencia el vínculo con los padres (Leidy, Guerra, \& Toro, 2012; Maddio \& Ison, 2013), con los maestros (Canto y Rodríguez, 1998) y con el vecindario o el entorno social próximo (Dupéré, Leventhal, \& Vitaro, 2012), tendrían una relevancia especial.

La evidencia científica señala que las creencias de autoeficacia en la niñez y adolescencia están asociadas a buenos resultados en la escuela. Las investigaciones de Gómez y Soares (2013), de Oliveira y Soares (2011) y Souza y Brito (2008) comprobaron que el rendimiento escolar de los niños y adolescentes brasileños, entre los 8 y los 14 años de edad, se relacionaba positivamente con la autoeficacia. Esto se debe, probablemente, a que los menores autoeficaces suelen tener mayor motivación intrínseca para el estudio (Martinelli, Grecci, \& Sassi, 2010), y considerarse más capaces de administrar eficazmente su proceso de aprendizaje (de Oliveira, \& Soares, 2011), mientras que la baja autoeficacia se vincula con problemas académicos y de comportamiento en la escuela (Medeiros, Loureiro, Linhares, \& Marturano, 2003). En esta misma línea, los estudios de Lescano (2008) en Argentina, y los de García Fernández et al. (2014) en Chile, mostraron que la autoeficacia académica de adolescentes entre 13 y 17 años de edad predecía significativamente: (a) la actitud y el interés hacia el estudio, (b) la motivación, (c) la administración del tiempo, (d) la concentración y la atención, y (e) la selección de ideas principales, entre otros hábitos y estrategias de estudio. Una investigación similar, pero realizada en niños argentinos de 8 a 12 años, también encontró que a medida que se 
incrementaba la autoeficacia académica aumentaba la motivación para el estudio y disminuían las dificultades de aprendizaje (Matus, 2007).

Con respecto al segundo estudio, los análisis de regresión logística revelaron que la autoeficacia académica percibida predijo positiva y significativamente los altos niveles de actitud, motivación, gestión del tiempo, concentración, procesamiento de la información, selección de ideas principales, ayuda al estudio, autoevaluación y estrategias de evaluación.

Además del desempeño escolar, en la infancia cobra fundamental importancia el desarrollo social y afectivo, ámbito donde la autoeficacia tiene también un rol crucial. En estudios locales se ha demostrado que los niños y adolescentes socialmente autoeficaces evitan utilizar modos de afrontamiento disfuncionales como el descontrol emocional y la paralización (Oros, 2004a), exhiben menos conductas transgresoras e impulsivas con sus pares y adultos (Ramirez, 2005), y experimentan mayor alegría, sentido del humor, optimismo y gratitud (Castillo, 2014). En contraposición, Cruvinel y Boruchovitch (2009), analizando una muestra de niños brasileños con sintomatología depresiva, encontraron que tenían en común bajas puntuaciones de autoeficacia en comparación con una muestra control. Estos mismos resultados fueron encontrados más recientemente por Galicia-Moyeda, SánchezVelasco y Robles-Ojeda (2013) en niños y adolescentes mexicanos de entre 12 y 15 años; por lo que podría pensarse que la autoeficacia actúa como un protector de problemas internalizantes.

También con relación al funcionamiento socioafectivo, una investigación española demostró que la autoeficacia de niños y adolescentes, entre los 8 y los 15 años de edad, se relaciona positiva y significativamente con la responsabilidad personal y social, indicando que a medida que se incrementan las creencias de autoeficacia aumenta el sentido de obligación moral respecto a uno mismo y a los demás (Sanmartín, Carbonell, \& Baños, 2011).

Otro ámbito en el que las creencias de autoeficacia durante la niñez y la adolescencia son de gran importancia, es el de la actividad física. Los programas cada vez más frecuentes de iniciación infantil deportiva y recreativa, y la Revista Iberoamericana de Diagnóstico y Evaluación - e Avaliação Psicológica. RIDEP · No44 · Vol.2 $172-181 \cdot 2017$ formación deportiva escolar, han despertado el interés de los psicólogos por analizar qué factores personales pueden predecir el éxito y el buen desempeño de los niños en actividades deportivas y lúdicas (Rojas, 2007), sin embargo los estudios empíricos en esta línea siguen siendo muy escasos. Algunos hallazgos permiten afirmar que la autoeficacia está positivamente asociada a la actividad física de niños y adolescentes, aumentando la motivación y el interés por realizar estas prácticas, incluso cuando son realizadas de manera voluntaria para mejorar el estado de salud (Dishman, et. al, 2004; Motl, et al. 2002; Ulloa, Taylor, Serrano, Rangel, Reynaga-Estrada, Acosta, \& González, 2013).

Como es de esperar, hay evidencias de que varones y mujeres se diferencian en sus percepciones de eficacia según el ámbito de desempeño que se evalúe. Por ejemplo, se ha encontrado que las niñas o mujeres estudiantes aventajan a los varones en autoeficacia académica y aprendizaje autoregulado (Justo, Andrade, \& Milani, 2009; Oros, 2004b; Teixeira, 2008), aunque análisis más pormenorizados permiten observar que los niños se perciben más autoeficaces que las niñas para realizar tareas de matemáticas y computación (Vekiri \& Chronaki, 2008; Zimmerman \& Martinez-Pons, 1990; Ferla, Valcke, \& Cai, 2009; Pérez, Lescano, Heredia, Zalazar, Furlán, \& Martínez, 2011), mientras que las niñas informan mayor autoeficacia en expresión verbal (Pajares, Miller, \& Johnson, 1999; Pajares \& Valiante, 1999). Además, las niñas reportan mayor autoeficacia interpersonal y social que los varones (Pérez, Lescano, Heredia, Zalazar, Furlán, \& Martínez, 2011; Carrasco Ortíz \& del Barrio Gándara, 2002), en tanto que estos últimos muestran mayor autoeficacia orientada a situaciones deportivas y de riesgo (Carrasco Ortíz \& del Barrio Gándara, 2002; Wigfield, Eccles, MacIver, Reuman, \& Midgley, 1991).

En síntesis, lo revisado hasta el momento pone de manifiesto que las creencias en la propia capacidad difieren según el género y tienen un impacto significativo en las conductas y emociones que despliegan y experimentan los niños y adolescentes, constituyendo por tanto un recurso de gran potencial para la salud física y mental. Su evaluación confiable permite predecir 
el desarrollo de otros constructos y detectar falencias que pueden ser suplidas tempranamente.

En el ámbito local, Oros (2004b) propuso una escala multidimensional para evaluar las creencias de autoeficacia en la niñez, que incluye las tres dimensiones de desempeño antes descriptas (académica, social y deportiva) y cumple con los criterios exigidos de confiabilidad y validez (Oros, 2004b). Sin embargo, a pesar de su utilidad, hasta el momento no existen baremos que proporcionen un valor de criterio para interpretar los resultados de esta escala, siendo que esta información podría resultar de suma relevancia para futuras investigaciones. El presente estudio, por tanto, pretende llenar este vacío al aportar las normas nacionales para niños y niñas de 9 a 13 años de edad.

\section{Método}

\section{Participantes}

Se realizó un estudio descriptivo y transversal del que participaron 1271 niños escolarizados, de ambos sexos (643 niñas y 628 varones), entre 9 y 13 años de edad $(M=11.17 ; \quad D E=1.07)$, procedentes de diferentes provincias (Entre Ríos, Mendoza, Santa Fe, \& Buenos Aires) de la República Argentina con fuerte predominancia de la región Mesopotámica (69\% de la región Mesopotámica; $19 \%$ de la región Cuyana, y 12\% de la región Pampeana). Según el tipo de institución educativa a la que asistían, los escolares pertenecían al nivel socioeconómico medio.

\section{Instrumento}

La EMA-i (Oros, 2004b) permite detectar cuán capaces o eficientes se consideran los niños para desempeñarse satisfactoriamente en tres de las áreas de funcionamiento más relevantes en la infancia (Harter 1982): la académica, la social y la deportiva. Se trata de una escala de autoinforme, constituida por 18 ítems (6 por cada dimensión) con un formato de respuesta tipo Likert de tres opciones: Si, Más o menos, y No. Los ítems expresan frases afirmativas en primera persona, presentan una longitud adecuada y describen un solo comportamiento a la vez. La escala es de sencilla aplicación, corrección e interpretación.
La validación inicial fue realizada por Oros (2004b) con una muestra de 620 niños argentinos escolarizados, comprendidos en un rango de edad de 8 a 13 años, pertenecientes al nivel socioeconómico medio. Todos los componentes de la escala resultaron discriminativos, factorialmente simples, unipolares y con cargas factoriales adecuadas, agrupándose con sentido psicológico y teórico en tres factores. La consistencia interna de la prueba completa, medida a través del coeficiente alpha de Cronbach, resultó satisfactoria (.81), como así también la de sus tres dimensiones: académica (.76), social (.74) y deportiva (.73).

\section{Procedimientos}

La muestra fue seleccionada de un modo no probabilístico, por conveniencia, según las posibilidades de acceso a los establecimientos educativos que brindaron su aprobación para realizar la administración de la prueba dentro del predio escolar. Los datos se recolectaron en más de 15 establecimientos educativos de nivel primario, de estrato predominantemente medio, ubicados en zonas urbanas de diferentes ciudades de cuatro provincias argentinas (Libertador San Martín, Diamante y Gualeguachú de la provincia de Entre Ríos; Santa Fe, de la provincia de Santa Fe; Mendoza, de la provincia de Mendoza; y Polvorines, de la provincia de Buenos Aires). En cada escuela, los niños completaron voluntariamente la escala, de forma colectiva en el horario habitual de clases, previa autorización escrita de sus padres o tutores legales. Las respuestas fueron procesadas mediante el programa estadístico SPSS. Para obtener la puntuación de cada niño se asignó un valor de 3 a la respuesta afirmativa, un valor de 2 a la respuesta intermedia y un valor de 1 a la respuesta negativa. A los ítems 2, 5, 8, 9, 6, 11, 12, 14, 15, 16 y 17; les correspondió la puntuación inversa. A continuación se sumaron los ítems correspondientes a la escala completa y a cada una de las tres dimensiones para obtener los puntajes brutos de autoeficacia académica, social y deportiva, respectivamente. Se realizaron análisis comparativos (ANOVAs y MANOVAs) entre los diferentes grupos de edad y sexo, y de acuerdo a estos resultados, y cuando correspondió, se computaron segregadamente los valores 
percentilares, las medias aritméticas y desvíos estándar de autoeficacia. Para estudiar la influencia de la edad, se seleccionaron intencionalmente dos subgrupos de niños: de 9 a $11(n=500)$ y de 12 a 13 años $(n=500)$, con el fin de lograr submuestras de comparación equivalentes en número y en períodos evolutivos similares.

\section{Resultados}

No se observaron diferencias significativas en el puntaje general de autoeficacia entre los diferentes sexos $(F \quad(1.1269)=2.86 ; p=.09)$ y grupos de edad (F $(1,995)=.07 ; p=.79)$. El promedio obtenidos por la muestra completa fue de 42.89 puntos $(\mathrm{DE}=.16)$. Los valores mínimo y máximo obtenidos por los niños fueron 21 y 54, respectivamente. Ningún niño obtuvo la puntuación mínima posible (18 puntos), mientras que 17 niños (1,3\%) alcanzaron el valor máximo permitido (54 puntos).

Cuando se realizaron análisis comparativos incluyendo las tres dimensiones de autoeficacia, se observaron diferencias significativas tanto en función del sexo $(F$ (3.1267) de Hotelling=3.03; $p=.029) \quad$ como de la edad $(F(3,992)$ de Hotelling $=25.09 ; p<.001)$. Las niñas presentaron valores más elevados que los varones en las dimensiones académica $(F \quad(1.1269)=5.08$; $p=.024)$ y social $(F(1.1269)=4.55 ; p=.033)$. Con relación a las edades, el grupo de 9 a 11 años aventajó a los niños mayores en la dimensión deportiva $(F(1.994)=9.75 ; p=.002)$, mientras que los niños de 12 a 13 años obtuvieron promedios más elevados en la dimensión social $(F$ (1.994) $=32.05 ; p<.001)($ Ver Tabla 1$)$.

En vista de estos resultados se calcularon los valores de referencia para la interpretación del puntaje general de autoeficacia, a partir de la muestra completa (ver Tabla 2), y se propusieron los criterios normativos de las dimensiones académica, social y deportiva, discriminados según sexo y edad (ver Tablas 2, 3 y 4).

\section{Discusión y Conclusión}

La construcción de baremos que permitan la interpretación diagnóstica de ciertas fortalezas y deficiencias psicológicas en el período de la niñez resulta de gran valor para el área clínica y el ámbito de la investigación epidemiológica. La interpretación fiable mejora la capacidad de decisión al momento de aplicar programas de intervención tendientes a fortalecer o modificar las creencias de los niños sobre sus propias capacidades de actuación.

El presente estudio, viene a llenar un vacío existente, al aportar las normas argentinas de la EMA-i que permitirán distinguir con precisión el nivel de autoeficacia para diversos ámbitos de actuación de niños y niñas escolarizados, y aportar valores de referencia para contrastar los datos obtenidos por otros evaluadores en diferentes muestras de niños argentinos.

No se tiene conocimiento de otros trabajos similares que permitan contrastar nuestros datos, por lo que no es posible determinar si los niños argentinos presentan similares puntuaciones que los niños de poblaciones internacionales en las

Tabla 1. Medias y desvíos estándar de autoeficacia según el sexo y la edad de los niños

\begin{tabular}{lcccccc}
\hline \multirow{2}{*}{ Variables } & \multicolumn{5}{c}{ Autoeficacia } \\
\cline { 2 - 7 } & \multicolumn{2}{c}{ Académica } & \multicolumn{2}{c}{ Social } & \multicolumn{2}{c}{ Deportiva } \\
\hline Sexo & $M E$ & $D E$ & $M E$ & $D E$ & $M E$ & $D E$ \\
Varones & 13.52 & 2.55 & 14.79 & 2.76 & 13.68 & 2.76 \\
Niñas & $\mathbf{1 3 . 8 5}$ & 2.58 & $\mathbf{1 5 . 1 2}$ & 2.78 & 13.56 & 2.51 \\
Edad & & & & & & \\
9-11 años & 13.60 & 2.84 & 14.37 & 2.94 & $\mathbf{1 3 . 8 0}$ & 2.88 \\
12-13 años & 13.31 & 2.47 & $\mathbf{1 5 . 3 5}$ & 2.68 & 13.24 & 2.57 \\
\hline
\end{tabular}


Tabla 2. Valores de referencia para la EMA-i en la muestra general

\begin{tabular}{lcc}
\hline Valores & Interpretación & Rango \\
\hline $18-32$ & Deficiente & \\
$33-35$ & Baja & Rango bajo \\
$36-39$ & Medianamente baja & \\
$40-44$ & Moderada & Rango medio \\
$45-47$ & Medianamente alta & \\
$48-50$ & Alta & Rango alto \\
$51-54$ & Muy alta & \\
\hline
\end{tabular}

Nota. Los valores exactos son los que corresponden a los percentiles $5,10,25,50,75,90$ y 95 son $32,35,39,44,47$, 50 y 51 , respectivamente.

tres dimensiones evaluadas. Se espera que próximos estudios puedan brindar información complementaria al respecto.

Las variaciones de los puntajes de autoeficacia en función del sexo y la edad de los niños no han sido sorprendentes pero sí relevantes. Los resultados indican que las niñas desarrollan creencias de autoeficacia más sólidas que los varones en los ámbitos social y académico. En la dimensión deportiva, si bien los varones aventajaron a las niñas, la diferencia no fue significativa desde el punto de vista estadístico. Estos resultados pueden explicarse, al menos en parte, por las inclinaciones e intereses diferenciales que desarrollan niños y niñas, sustentados en un sistema de creencias estereotipadas acerca de los géneros, que implica que los varones tienden, y son reforzados a, ser competitivos, bruscos, inquietos, aventureros y a disfrutar de tareas que implican riesgos, mientras que las niñas son más bien cooperativas, cariñosas, intuitivas, emotivas y más interesadas por tareas relacionadas con la lectura, la escritura y las relaciones interpersonales (Olaz, 2003; Vianna \& Finco, 2009). La idea de que las creencias de autoeficacia, así como otras creencias acerca del self, están influidas por el género ha sido apoyada también por otros trabajos de investigación (Bandura et. al, 1999; Pintrich \& Schunk, 1996). Carrasco Ortíz y Del Barrio Gándara (2002), por ejemplo, realizaron una investigación con más de 500 niños y adolescentes españoles, entre los 8 y los 15 años de edad, encontrando que los varones se percibían más eficaces que las mujeres para desempeñarse y resistir en actividades deportivas y de riesgo, mientras que éstas informaban mayor autoeficacia social, aunque cabe señalar que estas últimas diferencias sólo fueron significativas para el grupo de niñas de entre 12 y 13 años.

Específicamente con respecto a la edad, en este estudio se observó que los niños mayores se perciben más eficaces para establecer vínculos sociales afectivos que los más pequeños. Parecería que la autoeficacia social se afianza con el transcurso del tiempo debido posiblemente a la adquisición de nuevas habilidades de interacción. Las experiencias exitosas que sirven como indicadores de la propia capacidad se irían acumulando a medida que los años pasan y, dado que el grupo social se amplía, los niños tendrían la oportunidad de apreciar el comportamiento de un número cada vez mayor de modelos, factores que contribuyen también a fortalecer la autoeficacia (Bandura et al., 1999). Llama la atención el descenso que se produce en el nivel de autoeficacia deportiva en los niños de 12-13 años. Es probable que, con la llegada de la pubertad, si bien las actividades deportivas sigan siendo una de las principales atracciones de los niños, la atención en relación a las capacidades personales esté fundamentalmente centrada en los vínculos interpersonales, ámbito de primordial interés en la pre-adolescencia. La declinación de la autoeficacia deportiva y académica a medida que los niños crecen es un dato que ha sido informado también en otros trabajos de investigación (Carrasco Ortíz y Del Barrio Gándara, 2002; Cole, Martin, Peeke, Seroczynski y Fier, 1999; Shiner, 2000), aunque en el caso de Carrasco Ortíz y Del Barrio Gándara (2002) también se observó una disminución de la autoeficacia social a medida que aumentaba la edad, lo cual no ocurrió en nuestro estudio ni en otros similares. En síntesis, es normalmente esperable una alteración de la autoeficacia en función de las demandas e intereses típicos de cada etapa evolutiva.

Finalizando con el análisis de este estudio, cabe remarcar una limitación que es importante tomar en cuenta. Hubiera sido deseable realizar un muestreo aleatorio, estratificado por regiones, con el fin de garantizar mayor validez externa de los resultados, sin embargo esto no resultó posible y 
Tabla 3. Valores normativos de la Escala Multidimensional de Autoeficacia infantil (EMA-i)

\begin{tabular}{|c|c|c|c|c|c|c|c|c|c|c|c|c|}
\hline \multirow{3}{*}{ Percentiles } & \multicolumn{4}{|c|}{ Autoeficacia Académica } & \multicolumn{4}{|c|}{ Autoeficacia Social } & \multicolumn{4}{|c|}{ Autoeficacia Deportiva } \\
\hline & \multicolumn{2}{|c|}{ Varones } & \multicolumn{2}{|c|}{ Niñas } & \multicolumn{2}{|c|}{ Varones } & \multicolumn{2}{|c|}{ Niñas } & \multicolumn{2}{|c|}{ Varones } & \multicolumn{2}{|c|}{ Niñas } \\
\hline & $9-11$ & $12-13$ & $9-11$ & $12-13$ & $9-11$ & $12-13$ & $9-11$ & $12-13$ & $9-11$ & $12-13$ & $9-11$ & $12-13$ \\
\hline 5 & 9 & 9 & 10 & 9 & 10 & 10 & 9 & 10 & 9 & 8 & 9 & 10 \\
\hline 10 & 10 & 10 & 11 & 10 & 11 & 12 & 11 & 12 & 10 & 10 & 10 & 10 \\
\hline 25 & 12 & 12 & 12 & 12 & 13 & 14 & 13 & 14 & 12 & 12 & 12 & 12 \\
\hline 50 & 14 & 13 & 14 & 13 & 16 & 16 & 15 & 16 & 14 & 13 & 14 & 13 \\
\hline 75 & 15 & 15 & 16 & 15 & 17 & 17 & 17 & 18 & 15 & 15 & 16 & 15 \\
\hline 90 & 17 & 17 & 18 & 17 & 18 & 18 & 18 & 18 & 17 & 17 & 17 & 16 \\
\hline 99 & 18 & 18 & 18 & 18 & 18 & 18 & 18 & 18 & 18 & 18 & 18 & 18 \\
\hline Medias & 13.50 & 13.38 & 14.16 & 13.30 & 14.93 & 15.23 & 14.82 & 15.64 & 13.76 & 13.22 & 13.67 & 13.38 \\
\hline Desvíos & 2.65 & 2.36 & 2.52 & 2.60 & 2.81 & 2.60 & 2.85 & 2.58 & 2.65 & 2.65 & 2.60 & 2.35 \\
\hline$N$ & 365 & 263 & 406 & 237 & 365 & 263 & 406 & 237 & 365 & 263 & 406 & 237 \\
\hline
\end{tabular}

Nota. Los valores mínimos y máximos posibles para cada dimensión de la escala son 6 y 18, respectivamente.

Tabla 4. Rango percentilares de la EMA-I según el sexo y la edad

\begin{tabular}{|c|c|c|}
\hline Dimensiones & Rango & Interpretación \\
\hline \multicolumn{3}{|l|}{ Dimensión Académica } \\
\hline $\begin{array}{l}\text { Varones de } 9 \text { a } 13 \text { y niñas } \\
\text { de } 12 \text { a } 13 \text { años }\end{array}$ & $\begin{array}{l}6 \text { a } 12 \text { puntos } \\
13 \text { a } 14 \text { puntos } \\
15 \text { a18 puntos }\end{array}$ & $\begin{array}{l}\text { Autoeficacia baja } \\
\text { Autoeficacia moderada } \\
\text { Autoeficacia elevada }\end{array}$ \\
\hline Niñas de 9 a 11 años & $\begin{array}{l}6 \text { a } 12 \text { puntos } \\
13 \text { a } 15 \text { puntos } \\
16 \text { a } 18 \text { puntos }\end{array}$ & $\begin{array}{l}\text { Autoeficacia baja } \\
\text { Autoeficacia moderada } \\
\text { Autoeficacia elevada }\end{array}$ \\
\hline \multicolumn{3}{|l|}{ Dimensión Social } \\
\hline $\begin{array}{l}\text { Varones y niñas de } 9 \text { a } 11 \\
\text { años }\end{array}$ & $\begin{array}{l}6 \text { a } 13 \text { puntos } \\
14 \text { a } 16 \text { puntos } \\
17 \text { a18 puntos }\end{array}$ & $\begin{array}{l}\text { Autoeficacia baja } \\
\text { Autoeficacia moderada } \\
\text { Autoeficacia elevada }\end{array}$ \\
\hline Varones de 12 a 13 años & $\begin{array}{l}6 \text { a } 14 \text { puntos } \\
15 \text { a } 16 \text { puntos } \\
17 \text { a } 18 \text { puntos }\end{array}$ & $\begin{array}{c}\text { Autoeficacia baja } \\
\text { Autoeficacia moderada } \\
\text { Autoeficacia elevada }\end{array}$ \\
\hline Niñas de 12 a 13 años & $\begin{array}{c}6 \text { a } 14 \text { puntos } \\
15 \text { a } 17 \text { puntos } \\
18 \text { puntos }\end{array}$ & $\begin{array}{l}\text { Autoeficacia baja } \\
\text { Autoeficacia moderada } \\
\text { Autoeficacia elevada }\end{array}$ \\
\hline \multicolumn{3}{|l|}{ Dimensión Deportiva } \\
\hline $\begin{array}{l}\text { Varones de } 9 \text { a } 13 \text { y niñas } \\
\text { de } 12 \text { a } 13 \text { años }\end{array}$ & $\begin{array}{l}6 \text { a } 12 \text { puntos } \\
13 \text { a } 14 \text { puntos } \\
15 \text { a } 18 \text { puntos }\end{array}$ & $\begin{array}{l}\text { Autoeficacia baja } \\
\text { Autoeficacia moderada } \\
\text { Autoeficacia elevada }\end{array}$ \\
\hline Niñas de 9 a 11 años & $\begin{array}{l}6 \text { a } 12 \text { puntos } \\
13 \text { a } 15 \text { puntos } \\
16 \text { a } 18 \text { puntos }\end{array}$ & $\begin{array}{l}\text { Autoeficacia baja } \\
\text { Autoeficacia moderada } \\
\text { Autoeficacia elevada }\end{array}$ \\
\hline
\end{tabular}


en su lugar se empleó un método no probabilístico, en función de la posibilidad de acceso a las escuelas que autorizaron su participación y la realización de las evaluaciones en su predio escolar. Se espera en un futuro estudio poder revalidar estos datos normativos con los obtenidos mediante un muestreo estratificado que resulte aún más representativo de las diferentes regiones de nuestro país. De todas formas, se cree que esta primera aproximación, puede resultar de utilidad para futuras investigaciones y promover el interés por la evaluación y el fortalecimiento de las creencias de autoeficacia en la niñez.

\section{Referencias}

Bandura, A. (1986). Social foundations of thought and action: A social cognitive theory. Englewood Cliffs, NJ, USA: Prentice-Hall.

Bandura, A. (1987). Pensamiento y Acción. Barcelona: Martínez Roca.

Bandura, A. (1997). Self-efficacy: The exercise of control. New York, NY, USA: W. H. Freeman \& Co, Publishers.

Bandura, A., Pastorelli, C., Barbaranelli, C., \& Caprara, G. V. (1999). Self-efficacy pathways to childhood depression. Journal of Personality and Social Psychology, 76(2), 258-269.

Brenlla, M. E., Aranguren, M., Rossaro, M. F., \& Vázquez, N. (2010). Adaptación para Buenos Aires de la Escala de Autoeficacia General. Interdisciplinaria, 27(1), 77-94.

Canto, \& Rodríguez, J. E. (1998). Autoeficacia y educación. Revista Educación y Ciencia, 2(18), 45-53.

Carrasco Ortíz, M., \& Del Barrio Gándara, M. V. (2002). Evaluación de la autoeficacia en niños y adolescentes. Psicothema, 14(2), 323-332.

Castillo, S. (2014). Emociones positivas y autoeficacia en adolescentes de 12-14 años de la Provincia de San Juan. Tesis de Licenciatura. Universidad Adventista del Plata, Entre Ríos, Argentina.

Cid, P., Orellana, A., \& Barriga, O. (2010). Validación de la escala de autoeficacia general en Chile. Revista médica de Chile, 138(5), 551-557.
Cole, D. A., Martin, J. M., Peeke, L. A., Seroczynski, A. D., \& Fier, J. (1999). Children's over-and underestimation of academic competence: A longitudinal study of gender differences, depression and anxiety. Child Development, 70, 459-473.

Costa, A. M. (2012). Auto-conceito e auto-eficácia em crianças/jovens institucionalizados. Tesis de Maestría. Universidad Fernando Pessoa, Porto, Brasil.

Cruvinel, M., \& Boruchovitch, E. (2009). Autoconceito e crenças de autoeficácia de crianças com e sem sintomatologia depressiva. Interamerican Journal of Psychology, 43(3), 586-593.

de Oliveira, M. B., \& Soares, A. B. (2011). Autoeficácia, raciocínio verbal e desempenho escolar em estudantes. Psicologia: Teoria $e$ Pesquisa, 27(1), 33-39.

Dishman, R. K., Motl, R. W., Saunders, R., Felton, G. Ward, D. S., Dowda, M., \& Patee, R. R. (2004). Self-efficacy partially mediate the effect of a school-based physical-activity intervention among adolescent girls. Preventive Medicine, 628-636.

Dupéré, V., Leventhal, T., \& Vitaro, F. (2012). Neighborhood processes, self-efficacy, and adolescent mental health. Journal of health and social behavior, 53(2), 183-198.

Ferla, J., Valcke, M., \& Cai, Y. (2009). Academic self-efficacy and academic self-concept: Reconsidering structural relationships. Learning and Individual Differences, 19(4), 499-505.

Flammer, A. (1999). Análisis evolutivo de las creencias de control. En Albert Bandura (Ed.): Auto-Eficacia: Cómo afrontamos los cambios de la sociedad actual. España: Desclée de Brouwer.

Galicia-Moyeda, I. X., Sánchez-Velasco, A., \& Robles-Ojeda, F. J. (2013). Autoeficacia en escolares adolescentes: $\mathrm{Su}$ relación con la depresión, el rendimiento académico y las relaciones familiares. Anales de psicología, 29(2), 491-500.

García Fernández, J. M., Inglés Saura, C. J., Vicent Juan, M., Gonzálvez Maciá, C., Pérez Sánchez, A. M., \& San Martín, N. L. (2014). Validación de la Escala de Autoeficacia Percibida Específica de Situaciones 
Académicas en Chile y su Relación con las Estrategias de Aprendizaje. Revista Iberoamericana de Diagnóstico y Evaluación - e Avaliação Psicológica, 41(1), 118-131.

Gomes, G., \& Soares, A. (2013). Diferencia de género con relación al desempeño académico en estudiantes de nivel básico. Alternativas en Psicología, 17(28), 106-118.

Harter, S. (1982). The Perceived Competence Scale for Children. Child Development, 53, 87-97.

Justo, U. V., de Andrade, E. R., \& Milani, R. G. (Octubre, 2009). Avaliação da auto-eficácia de crianças em idade escolar. Trabajo presentado en el VI Encontro Internacional de Producao Científica Cesumar. Centro Universitario de Maringá, Maringá, Brasil.

Larson, L. M., Pesch, K. M., Surapaneni, S., Bonitz, V. S., Wu, T. F., \& Werbel, J. D. (2014). Predicting graduation the role of mathematics/science self-efficacy. Journal of Career Assessment, 1, 1-11.

Leidy, M. S., Guerra, N. G., \& Toro, R. I. (2012). Positive parenting, family cohesion, and child social competence among immigrant Latino families. Journal of Family Psychology, 24(3), 252-260.

Lescano, M. (2008). Influencia de los estilos parentales y las creencias de autoeficacia, en las metas académicas y las estrategias de aprendizaje en adolescentes. Tesis de Licenciatura. Universidad Adventista del Plata, Entre Ríos, Argentina.

Maddio, S., \& Ison, M. (Agosto, 2013). Relación entre la percepción de pautas de crianza, habilidades cognitivas para solucionar problemas interpersonales, autoconcepto y autoeficacia. Trabajo presentado en la XIV Reunión Nacional y III Encuentro Internacional de la Asociación Argentina de Ciencias del Comportamiento, Córdoba.

Martinelli, S. D. C., \& Grecci Sassi, A. D. (2010). Relações entre autoeficácia e motivação acadêmica. Psicologia: Ciência e Profissão, 30(4), 780-791.

Matus, C. (2007). La influencia de las creencias de autoeficacia en el uso de estrategias de aprendizaje en niños de EGB II. Tesis de Licenciatura. Universidad Adventista del Plata, Entre Ríos, Argentina.

Revista Iberoamericana de Diagnóstico y Evaluación - e Avaliação Psicológica. RIDEP · N44 · Vol.2 $\cdot 172-181 \cdot 2017$
Medeiros, P. C., Loureiro, S. R., Linhares, M. B., \& Marturano, E. M. (2003). O senso de autoeficácia eo comportamento orientado para aprendizagem em crianças com queixa de dificuldade de aprendizagem. Estudos de Psicologia (Natal), 8(1), 93-105.

Motl, R. W., Dishman, R. K., Saunders, R. P., Dowda, M., Felton, G., Ward, D. S., \& Pate, R. R. (2002). Examining social-cognitive determinants of intention and physical activity among black and white adolescent girls using structural equation modeling. Health Psychology, 21(5), 459.

Olaz, F. O. (2003). Autoeficacia y diferencia de géneros: aportes a la explicación del comportamiento vocacional. Revista de psicología general y aplicada: Revista de la Federación Española de Asociaciones de Psicología, 56(3), 359-376.

Oros, L, B. (2004a). Incidencia de las prácticas parentales sobre las creencias y los modos de afrontamiento del niño. Tesis Doctoral. Universidad Nacional de San Luis, San Luis, Argentina.

Oros, L. B. (2004b). Evaluación de las creencias de autoeficacia en la infancia. Revista Psicodiagnosticar, 14, 75-87.

Pajares, F. (1996). Self-efficacy beliefs in academic settings. Review of Educational Research, 66,543-578.

Pajares, F., Miller, M. D., \& Johnson, M. J. (1999). Gender differences in writing selfbeliefs of elementary school students. Journal of Educational Psychology, 91(1), 50.

Pajares, F., \& Valiante, G. (1999). Grade level and gender differences in the writing selfbeliefs of middle school students. Contemporary Educational Psychology, 24(4), 390-405.

Pérez, E., Beltramino, C., \& Cupani, M. (2003). Inventario de Autoeficacia para Inteligencias Múltiples. Fundamentos teóricos y estudios psicométricos. Evaluar, 3, 36-60.

Pérez, E., \& Cupani, M. (2008). Inventario de Autoeficacia para Inteligencias Múltiples Revisado (IAMI-R). Revista Latinoamericana de Psicología, 40 (1), 47-58.

Pérez, E., Lescano, C., Heredia, D, Zalazar, P., Furlán, L., \& Martínez, M. (2011). Desarrollo y análisis psicométricos de un inventario de 
Autoeficacia para inteligencias múltiples en niños argentinos. Psicoperspectivas, 10(1), 169-189.

Pérez, E., \& Medrano, L. A. (2007). Inventario de Autoeficacia para Inteligencias Múltiples Revisado: Un estudio de validez de criterio. Revista Avances en Medición, 5, 105-114.

Pérez, E., Medrano, L., Mattus, J., \& Ayllón, S. (2008). Adaptación de escalas de autoeficacia para escritura y lengua. Perspectivas en Psicología y Ciencias Afines, 5, 86-92.

Pintrich, P., \& Schunk, D. (1996). Motivation in Education: Theory, Research and Applications. Nueva York: Prentice-Hall.

Ramirez, P. (2005). Nivel de autoeficacia y estilos parentales en niños con problemas de conducta. Tesis de Licenciatura. Universidad Adventista del Plata, Entre Ríos, Argentina.

Rojas, N. (2007). Implicaciones de la Autoeficacia en el rendimiento deportivo. Pensamiento psicológico, 3(9).

Sanjuán, P., Pérez, A., \& Bermúdez, J. (2000). Escala de autoeficiencia general: Datos psicometricos de la adaptación para población española. Psicothema, 12 (2), 509 - 513.

Sanmartín, M. Carbonell, A., \& Baños, C. (2011). Relaciones entre empatía, conducta prosocial, agresividad, autoeficacia y responsabilidad personal y social de los escolares. Psicothema, 23(1), 13-19.

Shiner, R. L. (2000). Linking childhood personality with adaptation: Evidence for continuity and change across time into late adolescence. Journal of Personality and Social Psychology, 28, 310-325.

Souza, L. F., \& Brito, M. R. (2008). Crenças de autoeficácia, autoconceito e desempenho em matemática. Estudos de Psicologia, 25(2), 193-201.

Texeira, M. O. (2008). A Escala Multidimensional de Auto-eficácia Percebida: Um estudo exploratório numa amostra de estudantes do ensino superior. Revista Iberoamericana de Diagnóstico y Evaluación - e Avaliação Psicológica, 25(1), 141-157.

Ulloa, E. J., Taylor, J., Serrano, A. M., Rangel, S. P., Reynaga-Estrada, P. R., Acosta, J. J. M., \& González, J. C. (2013). Autoeficacia y actividad física en niños mexicanos con obesidad y sobrepeso. Sport TK: Revista
Euroamericana de Ciencias del Deporte, 2(2), 69-75.

Usher, E. L., \& Pajares, F. (2009). Sources of selfefficacy in mathematics: A validation study. Contemporary Educational Psychology, 34(1), 89-101.

Vekiri, I., \& Chronaki, A. (2008). Gender issues in technology use: Perceived social support, computer self-efficacy and value beliefs, and computer use beyond school. Computers \& Education, 51(3), 1392-1404.

Vianna, C., \& Finco, D. (2009). Meninas e meninos na educação infantil: Uma questão de gênero e poder. Cadernos Pagu, 33, 265-283.

Wigfield, A., Eccles, J. S., MacIver, D., Reuman, D. A., \& Midgley, C. (1991). Transitions during early adolescence: Changes in children's domain-specific self-perceptions and general self-esteem across the transition to junior high school. Developmental Psychology, 27(4), 552.

Zimmerman, B. J., \& Martinez-Pons, M. (1990). Student differences in self-regulated learning: Relating grade, sex, and giftedness to selfefficacy and strategy use. Journal of educational Psychology, 82(1), 51. 